\title{
5 Research Square

\section{Double and Triple Burdens of Malnutrition Among Child-Mother Pairs in Ethiopia: Spatial and Survey Regression Analysis}

Bethelihem Tigabu Tarekegn ( $\sim$ tbetelhemt@gmail.com )

University of Gondar

Nega Tezera Assimamaw

University of Gondar

Kendalem Asmare Atalell

University of Gondar

Selam Fisiha Kassa

University of Gondar

Addis Bilal Muhye

University of Gondar

Masresha Asmare Techane

University of Gondar

Tewodros Getaneh Alemu

University of Gondar

Chalachew Adugna Wubneh

University of Gondar

Getaneh Mulualem Belay

University of Gondar

Tadesse Tarik Tamir

University of Gondar

Destaye Guadie Kassie

University of Gondar

Amare Wondim

University of Gondar

Bewuketu Terefe

University of Gondar

Mohammed Seid Ali

University of Gondar

Beletech Fentie

University of Gondar

Almaz Tefera Gonete 
University of Gondar

\section{Berhan Tekeba}

University of Gondar

\section{Bogale Kassahun Desta}

University of Gondar

Melkamu Tilahun Dessie

University of Gondar

Amare Demsie Ayele

University of Gondar

\section{Research Article}

Keywords: Double Burden, Triple Burden, Malnutrition, Under Five Children, Spatial Distribution, Hotspot, Survey Regression, Associated Factors, Ethiopia

Posted Date: January 31st, 2022

DOI: https://doi.org/10.21203/rs.3.rs-1214721/v1

License: (c) (1) This work is licensed under a Creative Commons Attribution 4.0 International License. Read Full License 


\section{Abstract \\ Background}

Evidences on double and triple burdens of malnutrition at household level among child-mother pairs is a key towards addressing the problem of malnutrition. In Ethiopia, studies on the double and triple burdens of malnutrition are scarce. Even though, there is a study on the double burden of malnutrition at national level in Ethiopia it has limitations. It doesn't assess the triple burdens at all and a few forms of the double burden of malnutrition among child-mother pairs. Therefore, this study aimed to determine the prevalence and associated factors of double burden and triple burden of malnutrition among child-mother pairs in Ethiopia.

\section{Methods}

A total sample of 7,624 child-mother pairs from the Ethiopian Demographic and Health Survey 2016 were included in the study. All analysis were performed considering complex sampling design. Anthropometric measurements and hemoglobin levels of children and anthropometric measurements of their mothers were considered primarily to compute double burden of malnutrition (DBM) and triple burden of malnutrition (TBM). Spatial analysis was applied to detect geographic variation of prevalence of double and triple burdens of malnutrition among EDHS clusters. Bivariable and multivariable binary survey logistic regression models were used to assess the factors associated with DBM and TBM.

\section{Results}

The overall weighted prevalence of DBM and TBM respectively were $1.8 \%(95 \% \mathrm{Cl}: 1.38-2.24)$ and $1.2 \%$ (95\% Cl: 0.83-1.57) among pairs of a child-mother in Ethiopia. Significant clusters of high prevalence of DBM and TBM were identified. In the adjusted multivariable survey logistic regression models, middle household economic status [AOR $=0.23,95 \% \mathrm{Cl}: 0.06,0.89$ ] as compared to the poor, average birth weight $[A O R=0.26,95 \% \mathrm{Cl}: 0.09,0.80]$ as compared to large birth weight and children aged 24-35 months [AOR=0.19,95\% Cl: $0.04,0.95$ ] as compared to 6-12 months were less likely to experience DBM. Average birth weight $[\mathrm{AOR}=0.26,95 \% \mathrm{Cl}: 0.09,0.80]$ as compared to large birth weight and children aged 24-35 months [AOR=0.19,95\% Cl: $0.04,0.95]$ as compared to 6-12 months were less likely to experience TBM.

\section{Conclusion}

There is low prevalence of DBM and TBM among child-mother pairs in Ethiopia. However, interventions tailored on geographic areas, wealth index, birth weight and child birth could help to control the emerging DBM and TBM at household level among child-mother pairs in Ethiopia. 


\section{Background}

Apart from having enough food, having access to varied sources of food can help ensure diet quality, so that diets are sufficient not only in calories but also in micronutrients [1]. An imbalance in the amount of calories, protein and/or other nutrients consumed is referred to as malnutrition which commonly encompasses either undernutrition, overnutrition or micronutrient deficiencies. Undernutrition is one form of malnutrition that commonly include being underweight, stunting, and wasting. Now a days, every country in the world suffers from at least one type of malnutrition. It is critical to prevent undernutrition and micronutrient deficiencies since they are risk factors for child death, poor development, and early adult mortality [2][3]. Moreover, overweight or obesity in women is positively associated with several adverse maternal and fetal consequences throughout the period of pregnancy, delivery and the postpartum [4, 5]. Understanding the double burden of malnutrition (DBM) and triple burden of malnutrition (TBM) might be critical to accomplishing the goal of eradicating childhood malnutrition.

The DBM is referred to as the coexistence of undernutrition and overnutrition in the same nations, communities, or families [6]. The co-existence of an overweight or obese mother with undernourished child in the same household is an essential issue $[7,8]$. There are many countries worldwide facing DBM [9]. The TBM, on the other hand, refers to the co-existence of micronutrient deficiencies, undernutrition and overnutrition $[10,11]$. Micronutrient deficiencies include inadequate consumption of vitamins and minerals. In our context, the TBM form can include undernourished child (underweight, stunted or wasted), child micronutrient deficiency (anemia, which is often due to iron deficiency), and maternal overnutrition (overweight or obese for one's height). It is one of the leading causes of disease worldwide, affecting every country, particularly low- and middle-income countries.

Globally, child undernutrition is widespread and continues to be a major concern. Accordingly, an estimated $22.0 \%$, and $6.7 \%$ of children under five worldwide were stunted and wasted respectively [12]. Of which, most children with malnutrition live in Africa and Asia [12]. On the other hand, according to a recent review report in low- and middle income countries, $29.1 \%$ of children under the age of five years are stunted, $13.7 \%$ underweight, and $6.3 \%$ wasted from 2006-2018 [13]. Furthermore, undernutrition is responsible for roughly half of all fatalities among children under five. An estimated 6.3 million live-born children worldwide perished before the age of five due to undernutrition [14].

Despite the fact that Ethiopia continues to suffer with the burden of malnutrition, problems associated with DBM and/ or TBM are emerging as a public health concerns. When DBM is prevalent, it cannot be addressed only by overnutrition policies [15]. Maternal and child malnutrition in low-and-middle-income countries (LMIC) includes both undernutrition and the rapidly developing issues linked with overweight and obesity [16]. Inadequate intake of essential nutrients may weaken immune systems, hinder brain development, and increase the risk of conditions including anemia and blindness [17]. In this context, two billion individuals worldwide are affected by iron deficiency anemia.

Despite the fact that a number of public health interventions have been adopted in Ethiopia to address the various forms of malnutrition, the DBM and TBM yet, represent a new and major setback for 
Ethiopian nutrition policy. Ethiopia has made progress in eliminating hunger and, to a lesser extent, undernutrition; yet, malnutrition continues to be one of Ethiopia's primary public health challenges. As a consequence, the government adopted a National Nutrition Program, constructed an infant and young child feeding manual, and established a monthly child growth and monitoring program. However, it remains to be a serious public health issue in Ethiopia. The majority of research on child malnutrition in Ethiopia are descriptive, with just a few being analytical, and depending on pocket area survey data that may be difficult to generalize across varied Ethiopia. Even while there is a nationwide research study on the double burden of malnutrition in Ethiopia [18], it has limitations. It doesn't assess the triple burden of malnutrition at all and some forms of double burden of malnutrition among child-mother pairs.

As a result, the purpose of this study is to determine the prevalence and associated factors of the double burden and triple burden of malnutrition among child-mother pairs in Ethiopia. This work's evidence will assist policy makers, program designers and implementers in taking appropriate action to achieve sustainable development goal (SDG) 2030, which includes eradicating all forms of malnutrition in Ethiopia by 2030 .

\section{Methods}

\section{Study setting}

This study is a nationwide further analysis of Ethiopian Demographic and Health Survey (EDHS) data, which was collected between January 18, 2016 and June 27, 2016 [19]. The 2016 EDHS is the fourth national representative cross-sectional survey to be conducted as part of the global MEASURE DHS initiative, which is led by the Ethiopian Central Statistical Agency (CSA). The study's target population was pairs of all 6-59 month-old children with their mothers or caregivers in Ethiopia. Furthermore, the study population were pairs of all 6-59 month-old children with their respective mothers or caregivers in the randomly selected enumeration areas (EAs) of Ethiopia.

\section{Sampling procedures}

Briefly, the 2016 EDHS employed stratified, two-stage cluster sampling to identify the representative samples. The sampling frame for the 2016 EDHS consists of a total of 84,915 Enumeration Areas (EAs). In the first stage, 645 (202 urban and 443 rural) EAs were chosen. Figure 1 presents the map of survey cluster (EA) locations where raw dataset were collected. Then, in the second stage, a fixed number of 28 households was chosen from each enumeration area. A total of 16,650 households were included in the survey. A nationally representative population of 9504 children aged 6-59 months in the chosen households and a total of 15,683 women aged 15 - 49 years were interviewed with a $95 \%$ response rate. A thorough description of the survey design and sampling procedure can be found elsewhere [19].

The unit of analysis was the child-mother/care giver pair. the primary and secondary sampling units were clusters and households, respectively. To account for non-response, post-stratified weights were considered [20,21]. For selected survey years, sociodemographic and anthropometric data from children 
under the age of five and their mothers (aged 15-49 years) were retrieved. Hence, in this study, 7624 under-five children-mother/caregiver pairs with complete anthropometric and hemoglobin records were included.

\section{Variables of the study}

In this study, we looked at two binary outcome variables. Specifically, the double burden of malnutrition (DBM) and triple burden of malnutrition (TBM) among child- to- mother/caregiver pairs.

DBM among child - mother/caregiver- pairs was defined based on related literatures [22-25]. That is, $\mathrm{Yi}=1$ when a child is undernourished (either stunted, wasted, or under-weight based on WHO child growth standards [26]) and the mother/caregiver is over-nourished (overweight/obese), and $\mathrm{Yi}=0$ when neither is the case. Using the World Health Organization standards of BMI (weight (kg)/height (m2)) [27], maternal nutritional status was classified as underweight ( $\leq 18.4)$; normal (18.5-24.9); overweight (25.0-29.9); and obese $(\geq 30 \mathrm{~kg} / \mathrm{m} 2)$.

Based on related studies, the TBM was defined as a combination of the DBM of a child-mother pair plus an anemic child [22]. TBM includes undernourished children (either underweight, stunted, or wasted), an anemic child (with a micronutrient deficit, which is frequently caused by iron deficiency), and an overnourished mother/care-giver (with a weight higher than healthy for height, either overweight or obese).

The double and triple burden of malnutrition among child-mother pairs in Ethiopia is the result of a number of complicated, multifarious, and interconnected variables that work at several levels, one of which is maternal-related characteristics. Accordingly, the independent variables were selected based on literature and their availability in the data used. Household $(\mathrm{HH})$, mother and child characteristics are among the independent variables considered in this study. Specifically, residence (urban or rural) $[22,25$, $28]$, child age [22, 25, 28], HH wealth [22, 23, 25, 28], mother's age [22, 28], mother's education level [22, 23, $28]$, and child sex [22, 28]. Other socio-demographic and environmental factors include employment status, marital status, family size, source of drinking water, and type of toilet facility [22].

\section{Data processing and analysis}

The EDHS 2016 survey data sets and the Global Positioning System (GPS) points were obtained and processed with permission from Measure DHS (http://www.dhsprogram.com). The variables were then retrieved from the survey data for the children's data sets.

For spatial analysis, ArcGIS Pro version 2.4 was utilized, while R was used for the remaining analyses. Because of the nature the sampling design, all analyses were performed utilizing the complex sampling design adjustment approach and non-response rate. A survey package of $R$ [29] was used to estimate confidence intervals around prevalence by taking sample weights into account, which represent the inverse of the chance that the observation is included.

To estimate the prevalence of DBM and TBM across socio-demographic determinant variables, descriptive statistics were utilized. The prevalence of overweight/obese mother and stunted child 
(OM/SC), overweight/obese mother and wasted child (OM/WC), overweight/obese mother and underweight child (OM/UC), overweight/obese mother and anemic child (OM/AC), DBM, and TBM were presented as weighted percentages with $95 \%$ confidence intervals.

Further, the GPS coordinates were then combined with the prevalence of DBM and TBM in each of the EDHS 2016 clusters. As a result, the cluster level prevalence of DBM and TBM was exported into ArcGIS to depict hot and cold spots of clusters. Geographic variation in DBM and TBM prevalence among EDHS clusters was identified using spatial analysis $[30,31]$. Geographic variation of significant high prevalence or low prevalence of DBM and TBM was computed for each cluster using the Moran's I statistic [30]. Maps depicting the distribution and variations of DBM and TBM throughout the country were constructed. In addition, as a complement to Moran's I statistic, inverse distance weighted interpolation [31] was employed to estimate these distributions.

The standard binary logistic regression estimates are inadequate since the data originates from a complex survey design with stratification, clustering, and unequal weighting [32]. If the sampling design is not included in the analysis, the standard errors are likely be underestimated, perhaps leading to statistically significant findings when they are not $[20,21,32]$. As a result, the survey binary logistic regression model [32] was used to analyze data in order to account for the complex sampling design. The bivariate and multivariable survey binary logistic regression models were used to evaluate the associated factors of the DBM and TBM. Bivariate analysis was used to examine the relationship between sociodemographic characteristics and outcome variables. The multivariable analysis included all variables with $p$-value less than or equal to 0.25 in the bi-variable analysis. Variables with a p-value of $<0.05$ were considered statistically significant in multivariable analysis.

To demonstrate the strength of the association, the adjusted odds ratio (AOR) with the accompanying 95 percent confidence intervals were provided. These demonstrate whether each category of a variable raises $(A O R>1)$ or reduces $(A O R<1)$ the likelihood of having an DBM or TBM relative to the reference category, after adjusting for all other independent factors.

\section{Results}

\section{Characteristics of study participants}

We included 7,624 pairs of children aged 6-59 months and their mothers, with anthropometric and hemoglobin measurements. Weighted mean age of child in was 32 months (SD =15.75). Weighted median age of the mother/care giver was 29 years (IQR: 25-35). Weighted median household size was 6 (IQR: 4-7). Weighted median time to source of drinking water was 30 minutes (IQR: 10-60).

\section{Prevalence of malnutrition}

The weighted prevalence of child and maternal indicators are given (Table 1). The burden of anemia among children were major public health problem $(57.4 \%, 95 \% \mathrm{Cl}: 54.8,59.8)$ followed by stunting $(40.9 \%$, 
95\%Cl: 38.78, 42.95) in Ethiopia by 2016.

Table 1

Prevalence of nutritional status of children and mother's/caregiver's ( $\mathrm{n}=7624)$

\begin{tabular}{|l|ll|}
\hline \multirow{2}{|l|}{ Indicator } & Weighted Percent [95\% Cl] \\
\hline Child Stunting & $40.9[38.78,42.95]$ \\
\hline Child Wasting & $25.4[23.78,27.08]$ \\
\hline Child underweight & $9.4[8.27,10.51]$ \\
\hline Child anemia & $57.4[54.8,59.8]$ \\
\hline Maternal Overweight/Obesity & $5.8[4.94,6.59]$ \\
\hline
\end{tabular}

Specifically, 3.1\% (95\%Cl: $2.61-3.69)$ and $1.6 \%(95 \% \mathrm{Cl}: 1.18-1.99)$ of child-mother pairs experienced coexistence of overweight/obese mother and anemic child (OM/AC), and overweight/obese mother and stunted child (OM/SC) respectively (Table 2 ).

Table 2

The coexistence of various forms of malnutrition $(n=7624)$

\begin{tabular}{|ll|}
\hline Co-existence of malnutrition & Weighted Percent [95\%Cl] \\
\hline OM/AC & $3.1[2.61,3.69]$ \\
\hline OM/SC & $1.6[1.18,1.99]$ \\
\hline OM/WC & $0.8[0.49,1.04]$ \\
\hline OM/UC & $0.3[0.07,0.45]$ \\
\hline
\end{tabular}

The overall weighted prevalence of DBM and TBM respectively were $1.8 \%(95 \% \mathrm{Cl}: 1.38-2.24)$ and $1.2 \%$ (95\%Cl: 0.83-1.57) in Ethiopia (Table 3). The detailed prevalence of the indicators by factors also given. In the same table DBM and TBM by selected characteristics are given. Accordingly, prevalence of DBM among urban residents $(3.6 \%, 95 \% \mathrm{Cl}: 2.40-4.88)$ were higher than among rural residents $(1.6 \%$, $95 \% \mathrm{Cl}: 1.14-2.06)$. Furthermore, based on maternal age the highest prevalence of DBM $(2.7 \%, 95 \% \mathrm{Cl}: 1.45-$ 4.03) was observed among those in age range of 30-34 years old while the smallest prevalence $(1.1 \%$, $95 \% \mathrm{Cl}: 0.29-1.83)$ in age group of $15-24$ years old.

On the other hand, prevalence of TBM among urban residents was $1.6 \%(95 \% \mathrm{Cl}$ : $0.76-2.39)$ whereas $1.2 \%$ (95\% Cl: 0.76-1.56) among rural residents. Furthermore, based on maternal age the highest prevalence of TBM (1.8\%, 95\% Cl:0.69-2.86) was observed among those in age range of 30-34 years old while the smallest prevalence $(0.9 \%, 95 \% \mathrm{Cl}: 0.16-1.55)$ in age group of $15-24$ years old. 
Table 3

Weighted prevalence of DBM and TBM by socio-demographic characteristics of the study participants

\begin{tabular}{|c|c|c|c|}
\hline Variables & & $\mathrm{BDM}[95 \% \mathrm{Cl}]$ & TBM $[95 \% \mathrm{Cl}]$ \\
\hline \multirow[t]{3}{*}{ Toilet facility } & Un Improved & $1.9[1.27,2.46]$ & $1.1[0.62,1.53]$ \\
\hline & Improved & $2.9[1.81,3.98]$ & $1.8[0.97,2.71]$ \\
\hline & Open Field & $1.5[0.70,2.22]$ & $1.2[0.48,1.96]$ \\
\hline \multirow[t]{4}{*}{ Mother's Age } & $15-24$ & $1.1[0.29,1.83]$ & $0.9[0.16,1.55]$ \\
\hline & $25-29$ & $1.5[0.87,2.17]$ & $1.1[0.52,1.63]$ \\
\hline & $30-34$ & $2.7[1.45,4.03]$ & $1.8[0.69,2.86]$ \\
\hline & $35-49$ & $1.9[1.10,2.73]$ & $1.1[0.44,1.77]$ \\
\hline \multirow[t]{3}{*}{ HH size } & $2-4$ & $1.6[0.65,2.50]$ & $1.3[0.42,2.24]$ \\
\hline & $5-7$ & $1.8[1.16,2.39]$ & $1.1[0.57,1.54]$ \\
\hline & $8+$ & $2.1[0.95,3.29]$ & $1.4[0.42,2.31]$ \\
\hline \multirow[t]{2}{*}{ Residence } & Urban & $3.6[2.40,4.88]$ & $1.6[0.76,2.39]$ \\
\hline & Rural & $1.6[1.14,2.06]$ & $1.2[0.76,1.56]$ \\
\hline \multirow[t]{3}{*}{ Education Mother's } & No Formal Education & $1.8[1.24,2.41]$ & $1.2[0.76,1.73]$ \\
\hline & Primary & $1.8[1.01,2.60]$ & $1.2[0.47,1.93]$ \\
\hline & Secondary/Higher & $1.7[0.55,2.79]$ & $0.7[0.21,1.16]$ \\
\hline \multirow[t]{4}{*}{ Time to water source (in minutes) } & On Premise & $2.8[1.77,3.85]$ & $1.7[0.80,2.61]$ \\
\hline & $<=30$ & $1.9[1.26,2.51]$ & $1.2[0.68,1.77]$ \\
\hline & $31-60$ & $1.2[0.47,1.83]$ & $1.0[0.32,1.61]$ \\
\hline & $>=61$ & $1.9[0.84,3.03]$ & $1.2[0.35,2.00]$ \\
\hline \multirow[t]{2}{*}{ Media exposure } & No & $1.7[1.18,2.14]$ & $1.2[0.79,1.66]$ \\
\hline & Yes & $2.5[1.32,3.70]$ & $1.1[0.43,1.72]$ \\
\hline \multirow[t]{5}{*}{ Wealth index } & Poorest & $1.8[0.77,2.80]$ & $1.5[0.47,2.43]$ \\
\hline & Poorer & $2.2[1.04,3.35]$ & $1.6[0.50,2.64]$ \\
\hline & Medium & $0.7[0.00,1.39]$ & $0.7[0.00,1.38]$ \\
\hline & Richer & $1.5[0.53,2.37]$ & $0.7[0.01,1.33]$ \\
\hline & Richest & $3.5[2.11,4.78]$ & $1.7[0.84,2.49]$ \\
\hline
\end{tabular}




\begin{tabular}{|llll|}
\hline Variables & & BDM $[95 \% \mathrm{Cl}]$ & TBM [95\%Cl] \\
\hline Marital status & Currently in union & $1.8[1.32,2.17]$ & $1.2[0.82,1.54]$ \\
\cline { 2 - 4 } & Never/formerly in union & $2.9[0.46,5.39]$ & $1.5[0.00,3.46]$ \\
\hline Child sex & Male & $2.1[1.42,2.81]$ & $1.5[0.85,2.13]$ \\
\hline Birth weight & Female & $1.5[0.95,2.00]$ & $0.9[0.47,1.32]$ \\
& Large & $1.3[0.70,1.94]$ & $1.0[0.45,1.54]$ \\
\hline Child age (months) & Average & $1.9[1.26,2.62]$ & $1.0[0.51,1.53]$ \\
\cline { 2 - 4 } & Small & $2.3[1.37,3.13]$ & $1.8[0.97,2.62]$ \\
\hline 6-12 & $0.6[0.00,1.22]$ & $0.6[0.00,1.21]$ \\
\hline T-23 & $1.5[0.45,2.57]$ & $1.1[0.14,2.12]$ \\
\hline Total & $24-35$ & $2.2[1.12,3.22]$ & $1.7[0.78,2.63]$ \\
\hline
\end{tabular}

\section{Spatial distribution of DBM and TBM}

We used Anselin Local Moran's I [30] to detect statistically significant DBM and TBM clusters and outliers in Ethiopia. The analytic output is divided into four primary groups: two for clusters and two for outliers. High-High $(\mathrm{HH})$ and Low-Low (LL) clusters highlight similar enumeration regions with high and low prevalence respectively. High-Low $(\mathrm{HL})$ and Low-High $(\mathrm{LH})$ identifies outliers with high prevalence surrounded by enumeration areas of low prevalence and vice versa. Plots given below show the results of cluster and outlier analysis via Anselin Local Moran's I as well as interpolation plots for DBM and TBM.

Figure 2 depicts a cluster of DBM high values (hot spots) and low values (cold spots). This plot revealed the occurrence of hot spots of high prevalence of DBM in western and northwest Ethiopia. TBM hotspots, on the other hand, were observed in the northern Ethiopia, northwest Ethiopia, west Ethiopia, southern Ethiopia and eastern Ethiopia (Figure 3).

The estimated prevalence of DBM and TBM using interpolation analysis were undergone showing existence of spatial variation in prevalence of DBM and TBM, which supported by the results of cluster analysis (Figure 4).

\section{Associated factors of double burden of malnutrition}

The likelihood of experiencing double burden of malnutrition among child-mother/caregiver pairs are middle level wealth index as compared to the poor [AOR=0.23,95\% Cl: $0.06,0.89]$, average birth weight as 
compared to large birth weight [AOR=0.26, 95\% Cl: $0.09,0.80$ ] and child age group of $24-35$ months as compared to 6-12 months [AOR=0.19, 95\%Cl: 0.04,0.95] (Table 4).

Table 4

Associated factors of DBM among child-mother pairs in Ethiopia

\begin{tabular}{|c|c|c|c|c|}
\hline \multicolumn{2}{|l|}{ Variables } & \multirow{2}{*}{$\begin{array}{l}\text { Coefficients } \\
-0.52\end{array}$} & \multirow{2}{*}{$\begin{array}{l}\text { S.E. } \\
0.457\end{array}$} & \multirow{2}{*}{$\begin{array}{l}\text { P-value } \\
0.2526\end{array}$} \\
\hline Toilet Facility & Open field & & & \\
\hline & Unimproved & 0.01 & 0.348 & 0.9738 \\
\hline \multirow[t]{3}{*}{ Mother's Age } & $25-29$ & 0.01 & 0.493 & 0.9775 \\
\hline & $30-34$ & 0.51 & 0.500 & 0.3114 \\
\hline & $35-49$ & 0.27 & 0.618 & 0.6583 \\
\hline \multirow[t]{2}{*}{ HH size } & $5-7$ & 0.01 & 0.450 & 0.9858 \\
\hline & $8+$ & 0.14 & 0.529 & 0.7977 \\
\hline Residence & Rural & -0.26 & 0.507 & 0.6062 \\
\hline \multirow[t]{2}{*}{ Education } & Primary & 0.57 & 0.564 & 0.3085 \\
\hline & No Formal & 0.58 & 0.653 & 0.3774 \\
\hline \multirow[t]{2}{*}{ Time To Water } & $<=30 \mathrm{~min}$ & 0.28 & 0.542 & 0.6071 \\
\hline & $31-60 \min$ & -0.02 & 0.585 & 0.9694 \\
\hline Media Exposure & Yes & 0.46 & 0.388 & 0.2389 \\
\hline \multirow[t]{4}{*}{ Wealth Index } & Poorer & -0.39 & 0.549 & 0.4738 \\
\hline & Middle & -1.48 & 0.700 & 0.0351 \\
\hline & Richer & -1.08 & 0.635 & 0.0909 \\
\hline & Richest & -0.18 & 0.665 & 0.7868 \\
\hline Marital status & Never In Union/divorced/ widowed/separated & 0.79 & 0.511 & 0.1230 \\
\hline Child sex & Female & -0.35 & 0.323 & 0.2837 \\
\hline \multirow[t]{2}{*}{ Birth Weight } & Average & -1.33 & 0.564 & 0.0188 \\
\hline & Small & 0.07 & 0.337 & 0.8316 \\
\hline \multirow[t]{4}{*}{ Child Age } & $13-23$ & 1.28 & 0.877 & 0.1446 \\
\hline & $24-35$ & 1.62 & 0.799 & 0.0427 \\
\hline & $36-47$ & 1.33 & 0.779 & 0.0885 \\
\hline & $48-59$ & 1.37 & 0.791 & 0.0830 \\
\hline
\end{tabular}




\section{Associated factors of triple burden of malnutrition}

The likelihood of experiencing triple burden of malnutrition among child-mother/caregiver pairs are time to water source (in minutes) of $<=30 \mathrm{~min}[\mathrm{AOR}=0.49,95 \% \mathrm{Cl}: 0.26,0.93]$ and $31-60 \mathrm{~min}[\mathrm{AOR}=0.41,95 \% \mathrm{Cl}$ : $0.19,0.89]$ as compared to On Premise and average birth weight as compared to large birth weight [AOR=0.20, 95\% Cl: 0.0448, 0.909] (Table 5). 
Table 5

Associated factors of TBM among child-mother pairs in Ethiopia

\begin{tabular}{|c|c|c|c|c|}
\hline Variables & & Coefficients & S.E. & $\begin{array}{l}\mathrm{P} \text { - } \\
\text { value }\end{array}$ \\
\hline \multirow[t]{2}{*}{ Toilet Facility } & Open field & -0.57 & 0.491 & 0.2450 \\
\hline & Unimproved & -0.25 & 0.419 & 0.5469 \\
\hline \multirow[t]{3}{*}{ Mother's Age } & $25-29$ & 0.16 & 0.473 & 0.7328 \\
\hline & $30-34$ & 0.39 & 0.506 & 0.4458 \\
\hline & $35-49$ & 0.10 & 0.797 & 0.8959 \\
\hline \multirow[t]{2}{*}{ HH size } & $5-7$ & -0.38 & 0.484 & 0.4272 \\
\hline & $8+$ & -0.34 & 0.607 & 0.5790 \\
\hline \multirow[t]{2}{*}{ Educ } & Primary & 0.81 & 0.619 & 0.1886 \\
\hline & No Formal & 0.85 & 0.733 & 0.2455 \\
\hline \multirow{3}{*}{$\begin{array}{l}\text { Distance to water } \\
\text { source }\end{array}$} & $<=30 \mathrm{~min}$ & -0.71 & 0.327 & 0.0305 \\
\hline & $31-60 \mathrm{~min}$ & -0.89 & 0.393 & 0.0237 \\
\hline & Poorer & -0.68 & 0.726 & 0.3437 \\
\hline \multirow[t]{3}{*}{ Wealth Index } & Middle & -1.13 & 0.792 & 0.1524 \\
\hline & Richer & -1.53 & 0.809 & 0.0587 \\
\hline & Richest & -1.31 & 0.788 & 0.0987 \\
\hline Child sex & Female & -0.47 & 0.468 & 0.3193 \\
\hline \multirow[t]{2}{*}{ Birth Weight } & Average & -1.60 & 0.768 & 0.0375 \\
\hline & Small & -0.35 & 0.393 & 0.3681 \\
\hline \multirow[t]{4}{*}{ Child Age } & $13-23$ & 0.92 & 0.961 & 0.3414 \\
\hline & $24-35$ & 1.32 & 0.847 & 0.1186 \\
\hline & $36-47$ & 0.64 & 0.847 & 0.4481 \\
\hline & $48-59$ & 0.64 & 0.892 & 0.4761 \\
\hline Marital & $\begin{array}{l}\text { Never In Union/divorced/ } \\
\text { widowed/separated }\end{array}$ & 0.62 & 0.610 & 0.3103 \\
\hline
\end{tabular}

Discussion 
This study identified the prevalence and spatial distributions of double burden of malnutrition and triple burden of malnutrition as well as their associated factors among child-mother pairs within the same household in Ethiopia.

In Ethiopia, the overall weighted prevalence of DBM was 1.8\% (95\% Cl: 1.38-2.24) which is lower than $6.6 \%$ in Nepal [22]. Specifically, overweight/obese mother and stunted child were the most prevalent DBM which is in agreement with a comparative study in Addis Ababa with rural district of Kersa [33] as well as in nationwide study in Nepal [18]. However, the magnitude of overweight/obese mother and stunted child, $23 \%$, reported by Eshete et al. [18] is much greater than the result in this study. This discrepancy is because of the denominator considered. Apart from DBM, the coexistence of obese/overweight mother and anemic showed the next highest prevalence which is in agreement with similar study [18]. The overall weighted prevalence of TBM (co-existence of DBM and anemic child) was $1.2 \%(95 \% \mathrm{Cl}$ : $0.83-1.57)$ in Ethiopia which is lower than $7.0 \%$ in Nepal [22].

Furthermore, there were identified hotspots of high prevalence of DBM as well as hotspots of high prevalence of TBM. This shows that in addition to the overall burden DBM and TBM in the country, there was spatial variation in the DBM and TBM. Accordingly, the hot spots of high prevalence of DBM were observed in western and northwest Ethiopia and hot spots of high prevalence of TBM were observed in the northern Ethiopia, northwest Ethiopia, west Ethiopia, southern Ethiopia and eastern Ethiopia. Hence, further detailed investigations on spatial attributes leading to different effects on DBM and TBM could be conducted.

Wealth status, birth weight and child age were the identified associated factors of DBM. Children from middle wealth status compared to poor wealth status ( $\mathrm{AOR}=0.23,95 \% \mathrm{Cl}: 0.06-0.89$ ) were less likely to experience DBM. Mothers from richest wealth status compared to poor wealth status (AOR $=2.46,95 \%$ Cl: 1.17-5.15) were more likely to experience DBM [18]. The occurrence of DBM could be due to the consumption of energy-dense food that leads to overweight/obesity among the mothers, while the energy dense foods are not sufficiently nutrient dense to provide the children with adequate nutrition, leading to undernutrition [8]. The DBM was more common in households with higher income [8]. As a result, more focus should be placed on delivering health information linked to the intake of energy dense foods to wealthy households.

Children with average birth weight as compared to large birth weight $[\mathrm{AOR}=0.26,95 \% \mathrm{Cl}: 0.09,0.80]$ were less likely to experience DBM in agreement with a similar study [34]. Furthermore, a child with average birth weight as compared to large birth weight $[A O R=0.20,95 \% \mathrm{Cl}: 0.0448,0.909]$ were less likely to develop TBM. In majority of observational studies, birth weight was used as a proxy for early nutritional status. Consistent evidence suggests that higher birth weight and better nutritional status in childhood have a strong positive influence on the lean body mass in later life. Overall evidences suggest that larger birth weight in early life is associated with a higher lean body mass in later life. On the other hand, a few studies that assessed the long-term impact of protein energy supplementation in early life on the later 
lean body mass indicate modest and inconsistent benefit. Nutrition interventions for tackling undernutrition should aim to increase the lean body mass to address the DBM and TBM.

A child from age group of 24-35 months as compared to 6-12 months [AOR=0.19,95\% $\mathrm{Cl}: 0.04,0.95]$ were less likely to experience DBM. This result is in agreement with other studies [18] showing children aged between 24 and 59 months old, are more likely to encounter DBM significantly than the 0 to 23 months. A result from another study conducted among households in Myanmar and Pakistani is in agreement with this result [35]. This may be due to the combination of the different reasons; even though breast feeding has protective effect on both childhood undernutrition and maternal obesity, it is less practiced among children 24-59 months.

Likewise, results this study showed a child from a household with time to water source (in minutes) of $<=30 \mathrm{~min}[\mathrm{AOR}=0.49,95 \% \mathrm{Cl}: 0.26,0.93$ ] and $31-60 \mathrm{~min}[\mathrm{AOR}=0.41,95 \% \mathrm{Cl}: 0.19,0.89]$ as compared to on premise were less likely to develop TBM. A similar study showed that children who lived on households with access to water were found to be less likely to suffer from thinness than those who had no access [36]. Access to an improved water source is found to be an indicator of the higher probability of safe water [37]. The literature documents that the contamination of drinking water may particularly jeopardize children's nutritional status by inhibiting their growth and health, leading to malnutrition [37].

\section{Limitations}

The cross-sectional nature in this study, whereby it may not explain the causal and temporal relationship of associated factors with DBM and TBM. There might also be recall bias during answering child characteristics.

\section{Conclusion}

There is low prevalence of double burden of malnutrition and triple burden of malnutrition among childmother pairs in Ethiopia. Interventions tailored on geographic areas, wealth index, access to drinking water, birth weight and child birth could help to control double and triple burdens of malnutrition among child-mother pairs in Ethiopia.

\section{Abbreviations}

ANC

Antenatal Care

AOR

Adjusted odds ratio

BMI

Body Mass Index

$\mathrm{Cl}$

Confidence interval 
COR

Crude Odd Ratio

CSA

Central Statistical Agency

DBM

Double Burden of Malnutrition

EDHS

Ethiopian Demographic and Health Survey

EAs

Enumeration Areas

SD

Standard Deviation

SNNPR

South Nations and Nationality of Peoples Republic

SPSS

Statistical Package for Social Sciences

SSA

Sub Saharan Africa

TBM

Triple Burden of Malnutrition

USAID

United States Agency for International Development

WHO

World Health Organization

\section{Declarations}

\section{Ethics approval and consent to participate}

This study is a further analysis based on EDHS 2016 data, thus no separate ethical approval was required. However, EDHS 2016 was performed in accordance with the standard ethical guidelines of the DHS program. Accordingly, the ethical clearance was obtained from the Ethiopian Health and Nutrition Research Institute (EHNRI) Review Board, the National Research Ethics Review Committee (NRERC) at the Ministry of Science and Technology, the Institutional Review Board of ICF International, and the CDC. Informed consent was also obtained from all participants where informed consent for children was obtained from their parents/guardians. The authors requested the Measure DHS by briefly stating the objectives of this analysis and access was granted to use the data

(http://dhsprogram.com/data/available-datasets.cfm). 


\section{Consent for publication}

The manuscript does not contain any individual person's data.

\section{Availability of data and materials}

The minimal data used during the current study would be shared upon reasonable request from the correspondence.

\section{Competing interests}

The author declares that he/she has no competing interests.

\section{Funding}

No external fund was received for this study.

\section{Authors' contributions}

BTT contributed in the conceptualization and designing of the study, preparation of proposal, data acquisition, data analyses, result interpretation, drafting and reviewing the manuscript. All other authors contributed in reviewing the proposal, data analysis, result interpretation, drafting the manuscript and critically reviewed the final manuscript. All authors read and approved the final manuscript.

\section{Acknowledgements}

Our sincere thanks go to MEASURE DHS program for granting us the permission to use DHS data.

\section{References}

1. Maxwell S, Smith M. Household food security: a conceptual review. Househ food Secur Concepts, Indic Meas. 1992;1:1-72.

2. Battista MC, Hivert MF, Duval K, Baillargeon JP. Intergenerational cycle of obesity and diabetes: How can we reduce the burdens of these conditions on the health of future generations? Vol. 2011, Experimental Diabetes Research. 2011.

3. Kimani-Murage EW, Kahn K, Pettifor JM, Tollman SM, Dunger DB, Gómez-Olivé XF, et al. The prevalence of stunting, overweight and obesity, and metabolic disease risk in rural South African children. BMC Public Health. 2010;10. 
4. Stubert J, Reister F, Hartmann S, Janni W. The risks associated with obesity in pregnancy. Dtsch Arztebl Int. 2018;115(16):276.

5. Biswas T, Townsend N, Magalhaes RJS, Islam MS, Hasan MM, Mamun A. Current Progress and Future Directions in the Double Burden of Malnutrition among Women in South and Southeast Asian Countries. Curr Dev Nutr [Internet]. 2019 Jul 1;3(7):nzz026. Available from: https://doi.org/10.1093/cdn/nzz026

6. World Health Organization. The Double Burden of Malnutrition Policy Brief. Geneva: World Health Organization. WHO/NMH/NHD/17; 2017.

7. Roemling C, Qaim M. Dual burden households and intra-household nutritional inequality in Indonesia. Econ Hum Biol. 2013;11(4).

8. Dieffenbach S, Stein AD. Stunted child/overweight mother pairs represent a statistical artifact, not a distinct entity. J Nutr. 2012;142(4).

9. WHO. The double burden of malnutrition. Policy brief: World Health Organization. 2017. 2017;

10. Pinstrup-Andersen P. Agricultural research and policy for better health and nutrition in developing countries: A food systems approach. In: Agricultural Economics. 2007.

11. Meenakshi J V. Trends and patterns in the triple burden of malnutrition in India. Agric Econ (United Kingdom). 2016;47.

12. UNICEF, WHO, Group, The World Bank Group. Joint Child Malnutrition Estimates: levels and trends in child malnutrition: key findings of the 2021 edition. Who. 2021;24(2).

13. Ssentongo P, Ssentongo AE, Ba DM, Ericson JE, Na M, Gao X, et al. Global, regional and national epidemiology and prevalence of child stunting, wasting and underweight in low- and middle-income countries, 2006-2018. Sci Rep [Internet]. 2021;11(1):5204. Available from:

https://doi.org/10.1038/s41598-021-84302-w

14. World Health Organization. UNICEF /WHO/ World Bank Group - Joint Child Malnutrition Estimates: Levels and Trends in Child Malnutrition: Key Findings of The 2020 Edition. World Health Organization. 2020.

15. Jayalakshmi R, Kannan S. The double burden of malnutrition: an assessment of "stunted child and overweight/obese mother (SCOWT) pairs" in Kerala households. J Public Health Policy. 2019 Sep;40(3):342-50.

16. Black RE, Victora CG, Walker SP, Bhutta ZA, Christian P, De Onis M, et al. Maternal and child undernutrition and overweight in low-income and middle-income countries. Vol. 382, The Lancet. 2013.

17. Hawkes C, Demaio AR, Branca F. Double-duty actions for ending malnutrition within a decade. Vol. 5 , The Lancet Global Health. 2017.

18. Eshete T, Kumera G, Bazezew Y, Marie T, Alemu S, Shiferaw K. The coexistence of maternal overweight or obesity and child stunting in low-income country: Further data analysis of the 2016 Ethiopia demographic health survey (EDHS). Sci African. 2020;9. 
19. ICF. Ethiopia Demographic and Health Survey 2016 [Internet]. Addis Ababa, Ethiopia: CSA and ICF ; 2017. Available from: http://dhsprogram.com/pubs/pdf/FR328/FR328.pdf

20. Kalton G, Flores-Cervantes I. Weighting methods. J Off Stat. 2003;19(2):81.

21. Holt D, Smith TMF. Post stratification. J R Stat Soc Ser A. 1979;142(1):33-46.

22. Sunuwar DR, Singh DR, Pradhan PMS. Prevalence and factors associated with double and triple burden of malnutrition among mothers and children in Nepal: evidence from $2016 \mathrm{Nepal}$ demographic and health survey. BMC Public Health [Internet]. 2020;20(1):405. Available from: https://doi.org/10.1186/s12889-020-8356-y

23. Patel R, Srivastava S, Kumar P, Chauhan S. Factors associated with double burden of malnutrition among mother-child pairs in India: A study based on National Family Health Survey 2015-16. Child Youth Serv Rev [Internet]. 2020;116:105256. Available from: https://www.sciencedirect.com/science/article/pii/S0190740920309683

24. Fongar A, Gödecke T, Qaim M. Various forms of double burden of malnutrition problems exist in rural Kenya. BMC Public Health [Internet]. 2019;19(1):1543. Available from: https://doi.org/10.1186/s12889-019-7882-y

25. Masibo PK, Humwa F, Macharia TN. The double burden of overnutrition and undernutrition in mother-child dyads in Kenya: demographic and health survey data, 2014. J Nutr Sci [Internet]. 2020/01/24. 2020;9:e5. Available from: https://www.cambridge.org/core/article/double-burden-ofovernutrition-and-undernutrition-in-motherchild-dyads-in-kenya-demographic-and-health-survey-data2014/3F1509968705A021A5316990470430A5

26. De Onis M. WHO Child Growth Standards based on length/height, weight and age. Acta Paediatr Int J Paediatr. 2006;95(SUPPL. 450).

27. (CDC) C for DC and P. BMI Classification. 2011.

28. Pomati M, Mendoza-Quispe D, Anza-Ramirez C, Hernández-Vásquez A, Carrillo Larco RM, Fernandez $G$, et al. Trends and patterns of the double burden of malnutrition (DBM) in Peru: a pooled analysis of 129,159 mother-child dyads. Int J Obes [Internet]. 2021;45(3):609-18. Available from: https://doi.org/10.1038/s41366-020-00725-x

29. Lumley AT. Package "survey": analysis of complex survey samples. R package version 3.31-5. R Doc. 2016;

30. Anselin L. Local Indicators of Spatial Association-LISA. Geogr Anal. 1995;27(2).

31. Bartier PM, Keller CP. Multivariate interpolation to incorporate thematic surface data using inverse distance weighting (IDW). Comput Geosci [Internet]. 1996;22(7):795-9. Available from: https://www.sciencedirect.com/science/article/pii/0098300496000210

32. Roberts G, Rao NK, Kumar S. Logistic regression analysis of sample survey data. Biometrika. 1987;74(1).

33. Bliznashka L, Blakstad MM, Berhane Y, Tadesse AW, Assefa N, Danaei G, et al. Household-level double burden of malnutrition in Ethiopia: A comparison of Addis Ababa and the rural district of Kersa. Public Health Nutr. 2021; 
34. Kulkarni B. Addressing the Double Burden of Malnutrition in Developing Countries: Need for Strategies to Improve the Lean Body Mass. Food Nutr Bull. 2018;39(2_suppl).

35. Anik Al, Mosfequr Rahman M, Mostafizur Rahman M, Ismail Tareque M, Nuruzzaman Khan $M$, Mahmudul Alam M. Double burden of malnutrition at household level: A comparative study among Bangladesh, Nepal, Pakistan, and Myanmar. PLoS One. 2019;14(8).

36. Modjadji P, Madiba S. The double burden of malnutrition in a rural health and demographic surveillance system site in South Africa: A study of primary schoolchildren and their mothers. BMC Public Health. 2019;19(1).

37. Liu E, Balasubramaniam D, Hunt AF. Does access to water matter? A study on the nutritional status of primary-aged children in India. J Public Heal (United Kingdom). 2016;38(4).

\section{Figures}

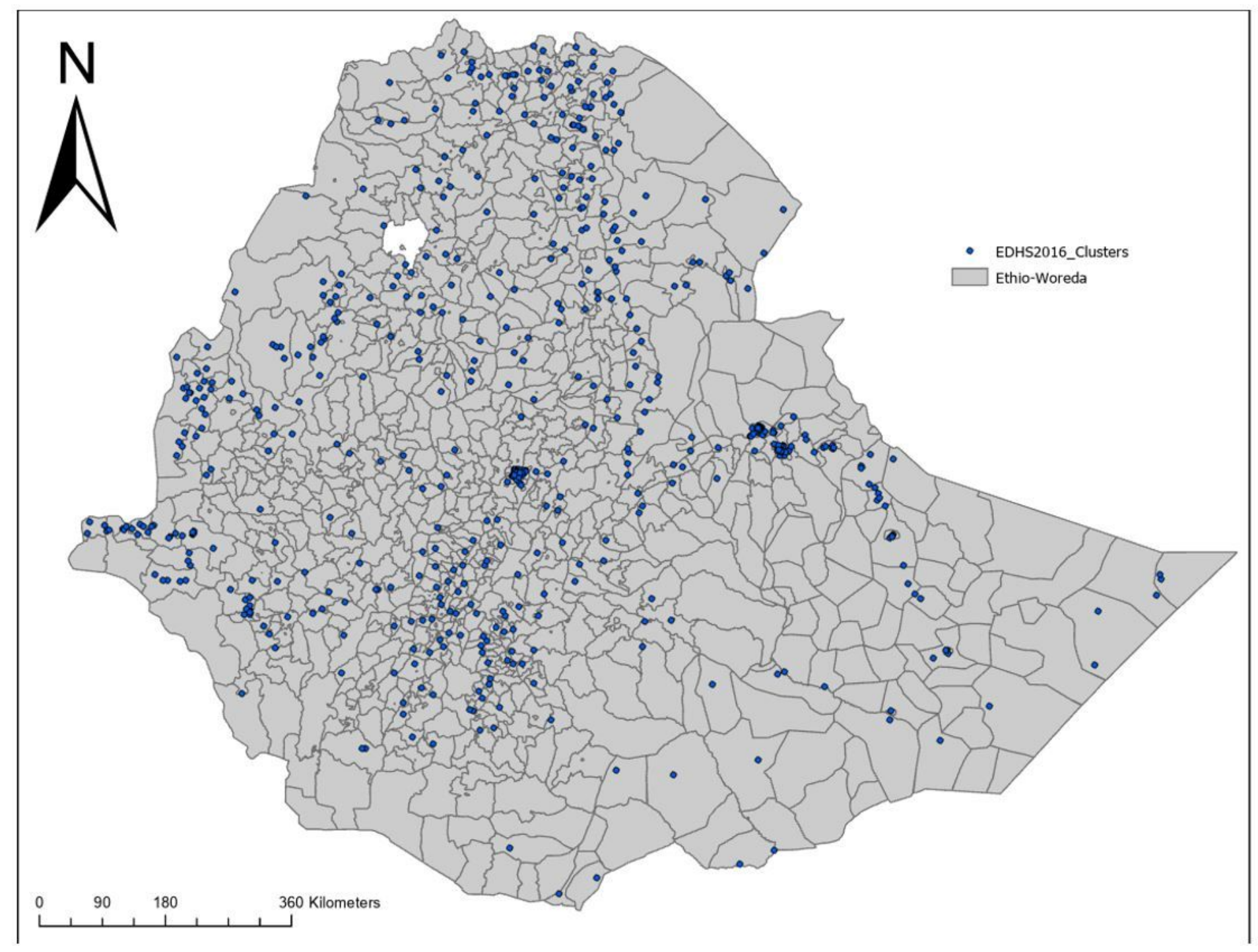




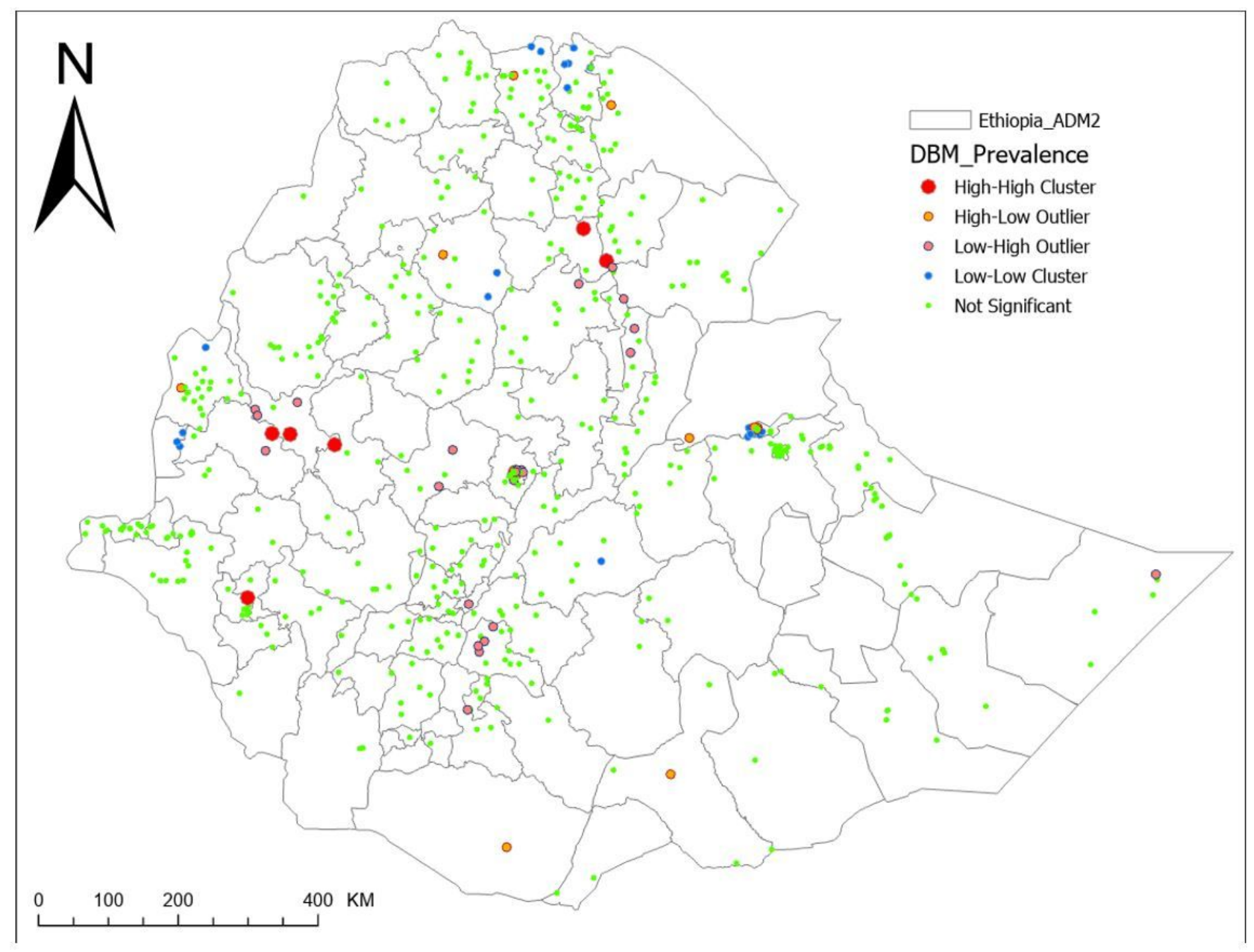

Figure 2

Pattern analysis (Geospatial Clustering and Hot Spot Detection) of double burden of malnutrition (DBM) among child-mother/caregiver pairs in Ethiopia 


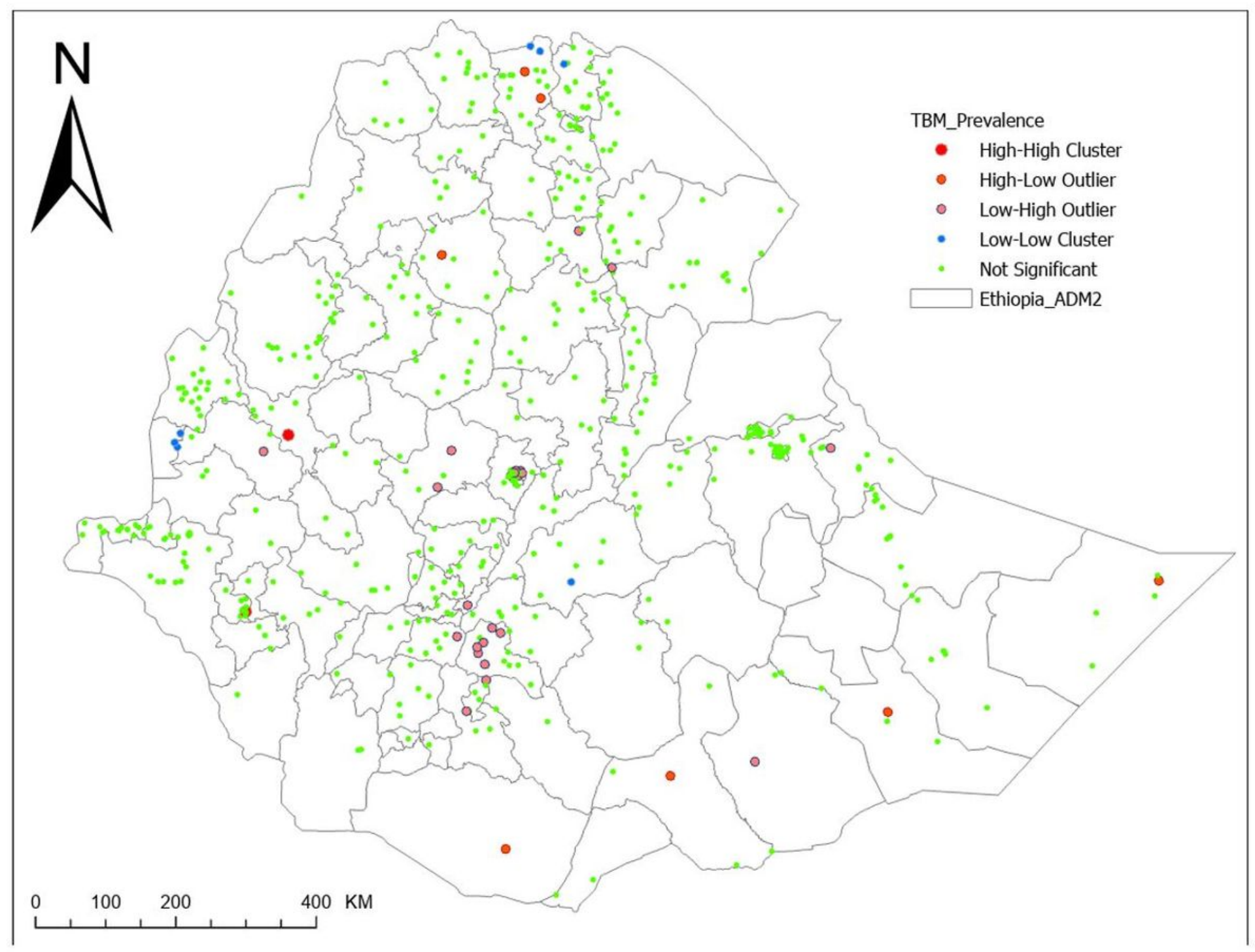

\section{Figure 3}

Pattern analysis (Geospatial Clustering and Hot Spot Detection) of triple burden of malnutrition (TBM) among child-mother/caregiver pairs in Ethiopia. 

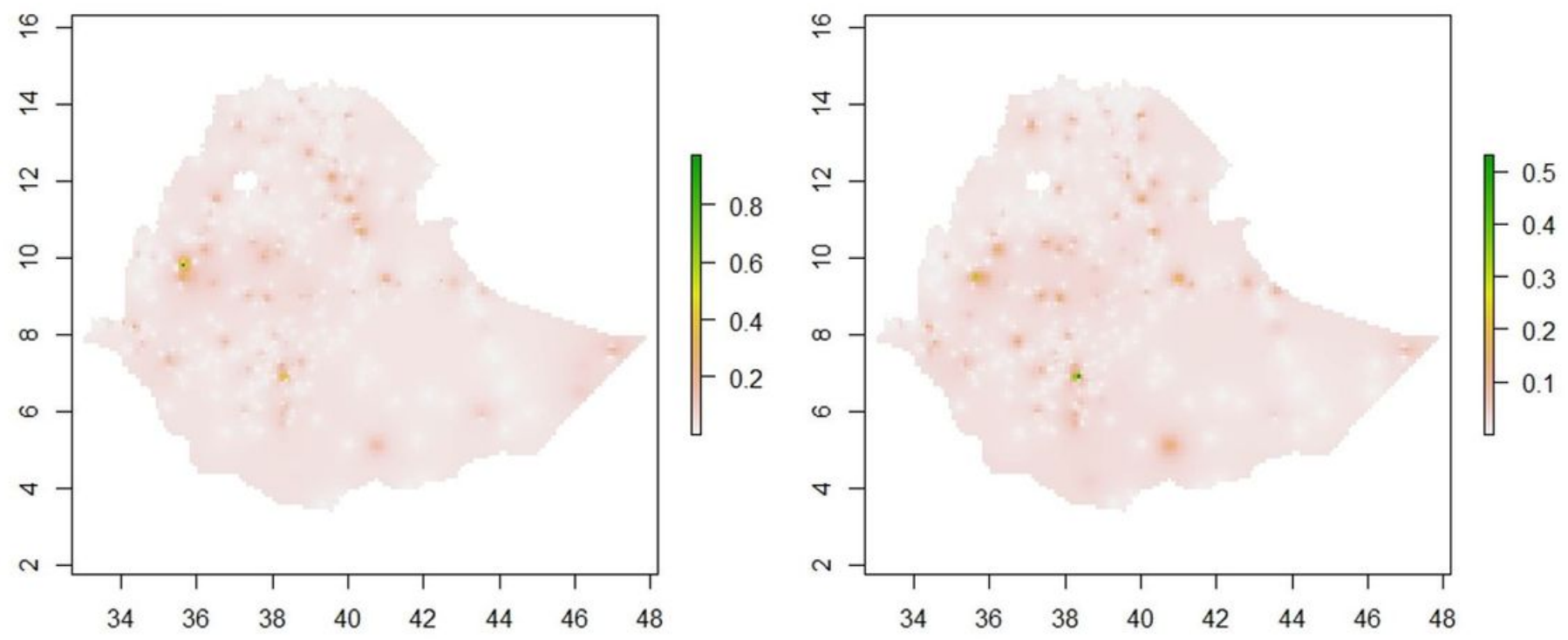

Figure 4

Spatial distribution for DBM (left side) and TBM (right side) via IDW interpolation. 